\title{
INTUBACIÓN Y MANEJO VENTILATORIO DEL PACIENTE CON CARDIOPATÍA AGUDA
}

\author{
INTUBATION AND VENTILATORY MANAGEMENT OF PATIENTS \\ WITH ACUTE HEART FAILURE
}

\author{
HERNÁN E. NÚÑEZ', LAURA BUIRA²
}

\section{RESUMEN}

Una de las formas de presentación más frecuentes de la insuficiencia cardíaca corresponde a la disnea, generalmente con hipoxemia. Resulta de capital importancia realizar un tratamiento rápido de sostén vital a fin de corregirla. Esto se puede lograr con oxigenoterapia, aunque es muy frecuente que además de esta los pacientes requieran asistencia ventilatoria mecánica, ya sea de forma invasiva como no invasiva.

El manejo apropiado de la vía aérea, la elección de la estrategia ventilatoria, así como las drogas a utilizar para sedoanalgesia en pacientes con falla cardíaca conducirán a obtener mejores resultados.

La siguiente revisión se enfocará en la asistencia ventilatoria mecánica invasiva en pacientes con enfermedad cardíaca aguda.

Palabras clave: edema pulmonar; insuficiencia cardíaca; respiración artificial; intubación.

\section{ABSTRACT}

One of the most frequent forms of presentation of heart failure corresponds to dyspnea, usually with hypoxemia. Itis of paramount importance to perform a rapid life support treatment in order to correct it. This can be achieved with oxygen therapy, although it is very common that in addition, these patients may require mechanical ventilation, either invasive or non-invasive. The appropriate management of the airway, the ventilation strategy, as well as the drugs for sedoanalgesia in patients with heart failure lead to the better results.

The following review will focus on invasive mechanical ventilatory assistance in patients with acute heart disease.

Keywords: pulmonary edema; heart failure; artificial respiration; intubation.

REVISTA CONAREC 2018;33(145):159-164 | DOI:10.32407/RCON/2018145/0159-0164

\section{INTRODUCCIÓN}

La falla cardiaca aguda se considera una patología que cursa con descompensación rápida del estado general del paciente y que se pone de manifiesto a través de la aparición de síntomas graves que requieren hospitalización. En esta circunstancia puede verse comprometido seriamente el aporte de oxígeno. En respuesta a esta hipoxemia se deben tomar medidas de sostén vital en forma rápida, como el uso de oxigenoterapia, drogas inotrópicas o vasopresoras y diuréticos. A pesar de estas intervenciones terapéuticas, la utilización del soporte ventilatorio mecánico es casi inevitable en la mayoría de las situaciones.

La disfunción ventricular aguda es una de las causas de indicación de asistencia ventilatoria mecánica (AVM) en pacientes hospitalizados. Las patologías cardiológicas más frecuentes asociadas

1. Médico Especialista en Terapia Intensiva. Médico interno de la UTI Hospital Juan A. Fernández CABA. Coordinador de Terapia Intensiva Sanatorio San Lucas, San Isidro, Buenos Aires. Coordinador UTI respiratoria Clínica Altergarten CABA. Instructor FCCS - SATl (Sociedad Argentina de Terapia Intensiva).

2. Médica Especialista en Terapia Intensiva. Médica de planta Sanatorio Suizo Argentino. Médica UTI Hospital Juan A. Fernández CABA. Jefa de trabajos prácticos del Departamento de docencia la SATI.

$\triangle$ Correspondencia:Dr. Hernán E.Núñez.Cerviño 3356, C1425.CABA. henunez@ gmail.com,

Los autores declaran no poseer conflictos de intereses.

Recibido: 02/04/2018 | Aceptado: 01/06/2018 a AVM son: edema agudo pulmonar (EAP), cardiopatía isquémico/ necrótica y posoperatorio de cirugía cardíaca central.

Los efectos cardiovasculares de la AVM en pacientes graves requieren un adecuado conocimiento para que las variables de la ventilación mecánica puedan ajustarse acorde a cada padecimiento y finalmente la desvinculación de la AVM se lleve a cabo cuando la causa que motivó la intubación esté resuelta.

Hay varios registros hospitalarios de falla cardíaca aguda en el mundo, como ADHERE (Acute Decompensated Heart Failure National Registry) y el Euro Heart Failure Surveys I y II, los cuales muestran un elevado porcentaje de pacientes con hospitalización prolongada asociada al uso de ventilación mecánica, y con datos que evidencian una importante tasa de mortalidad ${ }^{1,2}$.

El shock cardiogénico es una emergencia médica; el manejo clínico inicial se fundamenta en la rápida implementación de medidas de sostén vital, debiéndose asegurar la vía aérea con conexión del paciente a la ventilación mecánica, y además lograr un adecuado acceso vascular, con el objetivo de mejorar la hipoxia y reducir el consumo

Tabla 1. Factores de riesgo para ventilación mecánica invasiva en paciente con edema agudo pulmonar.

Edema alveolar difuso en la radiografía de tórax Infarto agudo de miocardio

Hipercapnia severa $\mathrm{pH}<7,25$

Presión arterial sistólica $<140$ mmHg al ingreso Fracción de eyección ventricular izquierda $<30 \%$ APACHE score elevado

APACHE score: Acute Physiology And Chronic Health Evaluation 
de oxígeno de los músculos respiratorios. Asimismo se debe reducir la pre- y la poscarga ventricular para optimizar la perfusión tisular³. El adecuado manejo de la vía aérea, la estrategia ventilatoria a seguir, así como la elección de las drogas a utilizar para sedoanalgesia en pacientes con shock cardiogénico merecen particular atención.

\section{DESARROLLO}

\section{MANEJO DE VÍA AÉREA Y DROGAS PARA INTUBACIÓN OROTRAQUEAL}

Una vez tomada la decisión de proceder a la intubación, siempre se debe contar con un plan alternativo en el caso de que fracase en la intubación u ocurra alguna complicación hemodinámica. El algoritmo de vía aérea difícil debe ser conocido y seguido, y el plan alternativo debe incluir la opción de despertar al paciente.

Es fundamental la adecuada preparación del paciente ${ }^{4}$.

De ser necesario, colocar sonda nasogástrica para evitar la aspiración del contenido gástrico, considerar eventualmente la utilización antiácidos, sean inhibidores de la bomba de protones o bloqueantes del receptor de histamina 2 .

Preoxigenar al paciente al menos 3 minutos con un flujo de oxígeno no menor a $10 \mathrm{l} / \mathrm{min}$. En pacientes obesos se recomienda realizar la preoxigenación en posición semisentado (cabecera a $25^{\circ}$ ) y se puede considerar la utilización de presión positiva no invasiva (CPAP: continuous positive airway pressure $)^{5}$.

No existe una droga o combinación ideal, ya que todas producen efectos secundarios asociados a su uso. Para reducir el riesgo de inestabilidad hemodinámica y complicaciones relacionadas con la vía aérea durante la intubación, se recomienda la combinación de un hipnótico y un opiáceo. También se puede utilizar un bloqueante neuromuscular.

En general, en situaciones de emergencia las drogas más utilizadas en nuestro medio son: propofol, midazolam, fentanilo, morfina, remifentanilo. Los bloqueantes neuromusculares pueden ser despolarizantes de la placa neuromuscular (succinilcolina) o no despolarizantes (atracurio, rocuronio).

El propofol produce descenso de la presión arterial y un aumento compensatorio del gasto cardíaco y del llenado ventricular, pero no así en la frecuencia cardíaca (efecto simpaticolítico y vagotónico).

Los efectos hemodinámicos del propofol son mucho más acentuados en los pacientes deshidratados (hipovolémicos) y en ancianos, así como en pacientes con compromiso ventricular izquierdo ${ }^{6}$.

Los efectos hemodinámicos del midazolam incluyen un moderado descenso de la presión arterial media (15 al 20\% con grandes dosis), del gasto cardiaco y del volumen sistólico.

A dosis terapéuticas usadas en la clínica, los opiáceos no producen depresión miocárdica importante y no predisponen a arritmias, pero pueden producir vasodilatación periférica, disminución de las resistencias sistémicas, e inhibir los reflejos barorreceptores, dando lugar a la aparición de hipotensión ortostática. En los enfermos coronarios, 8 a 15 mg de morfina administrado por vía intravenosa, producen una disminución en el consumo de oxígeno, de la presión de fin de diástole del ventrículo izquierdo y del trabajo cardíaco, pero el efecto sobre el índice cardíaco es poco significativo.
La succinilcolina, si bien suele ser segura dado su corta vida media, puede producir arritmias severas, dolor muscular, hiperkalemia y aumento de las presiones intraoculares e intragástrica. Se ha asociado a uso de esta droga la hipertermia maligna, poco frecuente, pero potencialmente fatal, con una incidencia de 1 en 50000 adultos.

Las drogas no despolarizantes tienen la capacidad de producir efectos sobre los receptores muscarínicos y los nicotínicos situados fuera de la unión neuromuscular. El atracurio y el vecuronio no poseen efectos cardiovasculares directos aunque el rocuronio tiene efecto vagolítico. La liberación de histamina puede llevar a vasodilatación, hipotensión y taquicardia compensadora, la mayoría de estos efectos ocurren con los compuestos bencilisoquinolínicos, en especial el atracurio.

El propofol a 2,5 mg/kg proporciona buenas condiciones para la intubación. Si se lo elige, debe utilizarse un opiáceo. La dosis de remifentanilo debe ser $4 \mathrm{mg} / \mathrm{kg}$ o superior, mientras que la dosis de fentanilo debe ser 3 a 5 ug/kg; esto produce un ligero efecto hipotensor, pero no inhibe completamente la respuesta al estímulo de la laringoscopia y la intubación; en este caso se recomienda asociar un bloqueante neuromuscular, como por ejemplo succinilcolina $1,5 \mathrm{mg} / \mathrm{kg}^{7}$ o rocuronio $0,6 \mathrm{mg} / \mathrm{kg}$. El midazolam puede utilizarse $(0,2-0,4 \mathrm{mg} / \mathrm{kg})$, teniendo en cuenta su efecto hipotensor, así como el aumento de las resistencias vasculares a los 3 minutos de su inyección?

\section{EVENTOS FISIOPATOLÓGICOS COMO CONSECUENCIA DE LA VENTILACIÓN MECÁNICA}

\section{EFECTOS CARDIOVASCULARES}

Los efectos cardiovasculares de la ventilación mecánica dependen de diversas variables incluyendo función miocárdica, volumen intravascular, presión intratorácica y tono autonómico intrínseco.

La circulación sanguínea está representada por dos compartimientos, uno dentro del tórax afectado por la presión intratorácica (PIT) y otro fuera del tórax afectado por la presión atmosférica, siendo así, los cambios generados por la ventilación mecánica o el esfuerzo respiratorio espontáneo son los que influyen en el compartimiento vascular intratorácico. El retorno venoso varía con la inspiración y espiración espontánea, y la presión del retorno venoso es influenciada por el volumen intravascular efectivo y su tono muscular, resultando así una presión venosa sistémica media, que tiene gradiente dinámico con la presión venosa central debido al movimiento diafragmático y los cambios de presión pleural. Durante la inspiración espontánea se reduce la PIT, permitiendo que la sangre fluya desde la periferia hacia las grandes venas intratorácicas y el corazón (la presión transmural de la aurícula derecha disminuye, aumentando el gradiente de presiones entre las venas cavas y la propia aurícula, facilitando el flujo sanguíneo o retorno venoso). El aumento inspiratorio del retorno sanguíneo venoso incrementa el volumen de fin de diástole del ventrículo derecho (o precarga) y secundariamente su volumen sistólico. Por el contrario, durante la ventilación mecánica con presión positiva, el aumento de la presión alveolar se transmite al espacio intrapleural y a todas las estructuras intratorácicas, induciendo un efecto de compresión de los grandes vasos que da lugar a un ascenso de la presión venosa central. Esta elevación de la presión auricular derecha reduce el gradiente de presión 
Tabla 2. Factores de riesgo asociados a la necesidad de ventilación mecánica.

- Edad >60 años.

- Sexo femenino

- Mayor estadía en la UTI.

- Localización anterior del IAM en mayor proporción.

- Grado Killip IV.

- Valor pico máximo de CPK > 2000.

- Reinfarto.

- Asociación de diabetes y HTA

UTI: unidad de terapia intensiva. HTA: hipertensión arterial. IAM: infarto agudo de miocardio CPK: creatinfosfoquinasa.

existente entre las venas sistémicas y el lado derecho del corazón, y se produce una reducción del retorno venoso y un descenso de la precarga ventricular derecha. Por otra parte, los cambios en el volumen pulmonar van a generar cambios sobre los vasos pulmonares, principalmente los alveolares, haciendo que durante la inspiración, al aumentar el volumen alveolar, la sangre contenida en los capilares se vea obligada a fluir hacia delante y hacia el final de la inspiración con presión positiva; si esta presión es alta, la compresión sobre los capilares aumenta la resistencia al flujo sanguíneo con las siguientes consecuencias: 1) disminución del vaciamiento ventricular derecho y aumento de la presión auricular derecha, y 2) la mayor resistencia al flujo sanguíneo disminuye el Ilenado auricular izquierdo y, por lo tanto, el volumen de fin de diástole ventricular izquierdo. Dependiendo de la volemia, el mayor volumen ventricular derecho puede desplazar el septum hacia la izquierda restringiendo aún más el llenado ventricular izquierdo y condicionando así un efecto de menor gasto cardíaco (GC), denominado interdependencia ventricular. Todo esto se traduce en disminución del gasto cardíaco e hipotensión arterial.

Asimismo, cuando se programan valores elevados de presión positiva de fin de espiración (PEEP) para evitar el colapso alveolar y optimizar la capacidad residual pulmonar, se incrementa la PIT y se reduce la precarga. La función ventricular derecha radica principalmente en mantener un adecuado volumen diastólico final, el cual está influenciado por la distensibilidad diastólica ventricular y la presión de distensión. La distensibilidad está disminuida en un ventrículo derecho dilatado, en isquemia aguda o sobredistensión del ventrículo izquierdo. Acorde a esto la estrategia ventilatoria que logra una reducción de las presiones para optimizar el retorno venoso son: disminuir el tiempo inspiratorio (Ti), incrementar el flujo inspiratorio (F) e incrementar la presión venosa sistémica media mediante la infusión de volumen isotónicos.

La poscarga ventricular derecha se define como el estrés de la pared durante la sístole, es decir que si aumenta la presión transmural se dilata el VD y disminuye su volumen-latido, debiendo incrementarse el volumen de fin de diástole para que esto no suceda. Por otro lado, el ventrículo izquierdo (VI) está fuertemente influenciado por la PIT, alterando el Ilenado ventricular y cambiando la poscarga del VI.

En pacientes isquémicos donde las presiones de llenado ventricular están adecuadas pero reducida su función miocárdica, la utilización de PEEP al disminuir la poscarga del VI mejora la función miocárdica. Cuando los pacientes son destetados de la ventilación mecánica, la pérdida súbita de la presión intratorácica, incrementa la poscarga nuevamente, disminuye el volumen de eyección del ventrículo izquierdo y con el incremento de las demandas metabólicas, en individuos susceptibles, puede causar isquemia, precipitar el desarrollo de edema pulmonar agudo e insuficiencia respiratoria aguda.
Además de la reducción del retorno venoso y el aumento de la resistencia vascular pulmonar, la ventilación mecánica puede conducir a disfunción ventricular isquémica. El flujo arterial coronario depende de la presión de perfusión coronaria (diferencia entre la presión diastólica aórtica y la presión telediastólica ventricular izquierda). La reducción de este gradiente de presión (disminución del gasto cardiaco, hipotensión arterial, aumento de la precarga) o la compresión de los vasos coronarios, como consecuencia del aumento de la presión intratorácica, pueden causar isquemia miocárdica.

\section{EFECTOS A NIVEL RENAL Y ENDOCRINO}

En un paciente con cardiopatía aguda, la ventilación mecánica con presión positiva reduce el gasto cardíaco, el cual se compensa por aumento de la frecuencia cardíaca y por aumento en las resistencias vasculares periféricas. A nivel renal, las células yuxtaglomerulares, altamente sensibles a la caída de presión, liberan renina, que actúa a nivel hepático formando angiotensina I y esta a su vez se transforma en angiotensina II, un vasoconstrictor potente. Estos fenómenos incrementan la presión arterial aun teniendo un menor gasto cardíaco.

Asimismo, el efecto de la presión positiva intratorácica sobre la presión arterial incrementa la liberación de hormona antidiurética (HAD), inhibiendo la libre excreción de agua y disminuyendo así la diuresis. Por otro lado, existen barorreceptores en las arterias carótidas y el arco aórtico que sensan la disminución de la presión inducida por la ventilación mecánica, resultando de un descenso de los niveles de HAD.

\section{BASES DE LA VENTILACIÓN MECÁNICA}

La AVM tiene como objetivo cumplir con dos funciones básicas: soporte ventilatorio en la falla cardiaca aguda y soporte en su oxigenación.

El soporte ventilatorio reemplaza total o parcialmente la función de los músculos respiratorios, disminuyendo el trabajo respiratorio inducido por el estado hipoxémico en el paciente cardiópata. Se logra mediante la implementación de presión positiva en la vía aérea, lo que permite entregar un volumen corriente apropiado y una frecuencia respiratoria similar a lo normal. Por otro lado, el soporte de la oxigenación se alcanza con suplemento en la fracción inspirada $\left(\mathrm{FiO}_{2}\right)$ y optimizando la relación ventilación perfusión con el fin de mejorar el intercambio del oxígeno a nivel alvéolo-capilar.

A través de la ventilación con presión positiva se busca restablecer la capacidad residual funcional, perdida por colapso alveolar y evitar la fatiga muscular de pacientes hipoxémicos con cardiopatía aguda. La disnea, en los pacientes con falla cardíaca aguda, incrementa el trabajo respiratorio de los músculos inspiratorios prolongando el tiempo inspiratorio y ocasionando fatiga muscular.

Existen dos factores que pueden ocasionar efectos hemodinámicos deletéreos: la magnitud de la presión inspiratoria y la duración de la misma. Ambos factores se combinan en un parámetro denominado presión media de la vía aérea; así, mientras menor sea el valor de este parámetro, menores serán los efectos hemodinámicos. 


\section{PROGRAMACIÓN DEL VENTILADOR}

Al programar un respirador, todas las variables deben ser ajustadas de acuerdo al monitoreo de la mecánica respiratoria, los gases arteriales y el estado hemodinámico del paciente.

Modo ventilatorio: se debe elegir aquella modalidad ventilatoria que mejor se adecue a las demandas metabólicas del paciente. Esto significa que en un paciente que esté cursando una cardiopatía aguda e insuficiencia respiratoria, ya sea por un proceso isquémico coronario con descompensación hemodinámica, arritmia descompensada (por ejemplo: fibrilación auricular de alta respuesta ventricular con hipotensión arterial) o insuficiencia cardíaca que no responde a tratamiento médico no invasivo, deberá optarse por un modo ventilatorio controlado ya sea por presión o por volumen. Esto dependerá del modo que logre la mejor sincronización del paciente y el respirador artificial.

Los parámetros que deben ser establecidos en el equipo de ventilación mecánica son:

Volumen corriente (VC) o volumen tidal: en cardiópatas sin afección pulmonar se calcula de 6 a $8 \mathrm{ml} / \mathrm{kg}$ de peso ideal del paciente, pudiendo modificarse en base a la estrategia ventilatoria planteada siempre controlando que la presión meseta o plateau de la vía aérea generada por el VC no supere los $30 \mathrm{CmH}_{2} \mathrm{O}$.

Frecuencia respiratoria (FR): los pacientes con cardiopatía avanzada tienen un trabajo respiratorio incrementado, por lo tanto su programación debe ser mayor a lo habitual, es decir 18-20 respiraciones minuto. No obstante debe adecuarse la programación de este parámetro a la $\mathrm{PaCO}_{2}$ deseada, y además verificar que no haya atrapamiento aéreo mediante la curva de flujo/tiempo o la cuantificación de auto-PEEP (presión espiratoria al final de la espiración positiva). Si es necesario, debe ajustarse la dosis de sedoanalgesia con opiáceos y benzodiazepinas para lograr la mejor la adaptación del paciente a ventilador.

Flujo inspiratorio: es la velocidad a la que ingresa el volumen programado en un tiempo determinado. La utilización de un flujo inspiratorio rápido permite el suministro del volumen circulante en un tiempo más corto (menor tiempo inspiratorio), lo cual produce una reducción de la presión media de la vía aérea en los pacientes con resistencias normales. Además, un flujo alto genera un aumento de la presión pico inspiratoria, y puesto que gran parte de esta presión se dispersa en las vías aéreas de conducción y no se transmite a los espacios alveolar e intrapleural, puede dar lugar a una ventilación desigual. En contraste, el uso de un flujo inspiratorio lento disminuye la presión pico, pero provoca un aumento de la presión media al generar atrapamiento aéreo (auto-PEEP). El objetivo debe ser la utilización de un flujo inspiratorio apropiado, ni demasiado rápido ni excesivamente lento, y lo establecido en estos pacientes es de 50-60 l/min, cuidando que la relación inspiración/espiración (I:E) sea la adecuada de 1:2 a 1:4. Valores superiores a 1:1 aumentarán de forma significativa la presión media de la vía aérea, generarán atrapamiento aéreo y acentúan la afectación hemodinámica.

Fracción inspirada de oxígeno: es aquella que permita alcanzar una adecuada saturación de oxígeno ( $\mathrm{SpO}_{2}$ entre 91 a 94\%), y debe evitarse el uso de fracciones mayores al 60\%, relacionadas con efectos tóxicos y daño alveolar (estrés oxidativo a nivel celular).
Presión positiva al final de la espiración (PEEP): habitualmente se ajus ta a $5 \mathrm{CmH}_{2} \mathrm{O}$ para compensar la pérdida de volumen de las regiones pulmonares posteriores colapsadas por el decúbito. En casos como el edema agudo pulmonar, se requieren mayores niveles de PEEP. Sensibilidad inspiratoria: en pacientes con cardiopatía severa, la sensibilidad óptima es aquella que permite al paciente disparar el respirador con el menor esfuerzo posible sin que exista autodisparo y se utiliza de -0.5 a $-2 \mathrm{CmH}_{2} \mathrm{O}$ en el disparo por presión y de 1-2 I/min en el disparo por flujo.

Las alarmas también son un elemento básico en la programación del ventilador y hay que vigilar su correcta programación y funcionamiento para disminuir la posibilidad de complicaciones graves

\section{AVM EN EDEMA AGUDO DE PULMÓN}

La claudicación aguda del ventrículo izquierdo en su función de bomba produce acúmulo de líquido en el espacio intersticial y los alvéolos pulmonares, lo cual incrementa el trabajo respiratorio, compromete el adecuado intercambio de gases, resultando en fatiga respiratoria e hipoxemia. Existen alteraciones sistémicas como hipotensión arterial, oliguria, incluso anuria, acidosis metabólica, signos de hipoperfusión periférica y trastornos neurológicos (agitación o deterioro del sensorio).

En el edema agudo pulmonar la ventilación mecánica no invasiva (VNI) proporciona soporte ventilatorio parcial que no necesita de una vía aérea artificial y que mejora el intercambio gaseoso y alivia la disnea. Además la VNI disminuye el trabajo respiratorio al disminuir la carga de los músculos inspiratorios y evita su fatiga. Tanto el CPAP como el BIPAP (bilevel positive airway pressure) disminuyen el trabajo respiratorio y previenen en un gran porcentaje las intubaciones en pacientes con edema agudo. En un estudio de 100 pacientes asignados aleatoriamente a recibir oxígeno suplementario vs. CPAP (3-12 $\mathrm{cmH}_{2} \mathrm{O}$ ), con todos los pacientes sometidos a cateterización pulmonar, se encontró que el grupo de pacientes con CPAP tuvo una mejoría en la $\mathrm{PaO}_{2}$ (presión arterial de oxígeno) asociado con una disminución significativa de shunt intrapulmonar, además de un mejor índice de GC y con menor incidencia de intubación traqueal (16\% en el grupo de (PAP vs. 36\% en el grupo de oxígeno suplementario) $)^{9,10}$.

El CPAP consiste en un sistema de entrega constante de presión en la vía aérea durante la inspiración y la espiración, evitando el colapso o cierre completo de las unidades alveolares y, al incrementar la presión alveolar, esta se hace mayor que la presión de los capilares pulmonares, trayendo como consecuencia disminución en la poscarga y mejoría del gasto cardíaco.

Los ventiladores no invasivos que disponen de la modalidad BiPAP emplean ciclos de alto flujo. En respiración espontánea los pacientes inician la inspiración mediante un esfuerzo voluntario; esta inspiración es detectada por el ventilador que le aporta un flujo determinado en un tiempo dado para lograr la presión inspiratoria en la vía aérea programada (IPAP); la espiración comienza cuando la velocidad de flujo inspiratorio disminuye a un 25\% del pico flujo máximo y durante la misma se mantiene una presión positiva espiratoria (EPAP) que logra una presión alveolar suficiente para impedir la formación de atelectasias, manteniendo como resultado una capacidad residual funcional normal. El modo BIPAP es un análogo cualitativo de ventilación con presión 
soporte (PSV) más PEEP, siendo la presión de soporte el resultante de la diferencia entre IPAP y EPAP, es decir, EPAP=PEEP. Esto provoca que toda modificación del EPAP sin cambio proporcional del IPAP Ileve a un aumento o disminución de la PSV.

La PSV en el edema agudo de pulmón se considera como una ventilación parcial, iniciada por el paciente, limitada por presión y ciclado por flujo. Se inicia con el esfuerzo inspiratorio espontáneo del paciente, el respirador presuriza el circuito y suministra un flujo inspiratorio alto. La velocidad de presurización y el flujo ajustan el tiempo que tarda en alcanzar una presión meseta. Durante el resto de la inspiración se administra un flujo desacelerado, establecido por el nivel de soporte, las propiedades mecánicas del sistema respiratorio y del esfuerzo inspiratorio. Para determinar el nivel adecuado de presión inspiratoria durante la PSV se han descrito diferentes métodos. MacIntyre utilizó el máximo nivel de PSV que resultara en una condición de patrón respiratorio estable, sin fases de hiper- o hipopnea. Brochard ha recomendado observar el nivel de reclutamiento de los músculos accesorios de la inspiración, principalmente el esternocleidomastoideo, a través de la inspección y palpación ${ }^{11}$. Diversos autores concuerdan, por otro lado, en que la frecuencia respiratoria es un modo sencillo y práctico para verificar el nivel adecuado de PSV, siendo valores entre 15-25 respiraciones/min los recomendados.

El éxito de la VNI no es fácil, sobre todo en los pacientes con disnea continua, debido al edema agudo pulmonar; la colocación de la interfase debe conseguir comodidad, tolerancia y eficacia, y puede ser mascarilla nasal, oronasal, facial total y sistema Helmet. Lo más recomendado en este tipo de pacientes son las mascarillas oronasales o la facial total, ya que reciben el flujo de gas por ambos orificios naturales y el efecto presión positiva del ventilador no se pierde. Los pacientes con edema pulmonar que no respondan adecuadamente con la VNI debido a una mala interacción paciente-ventilador tendrán que ser intubados para una ventilación mecánica invasiva bajo sedación. Estos pacientes están expuestos a un mayor riesgo de neumonía y tienen una mayor mortalidad (Tabla 1).

\section{AVM EN INFARTO AGUDO DE MIOCARDIO COMPLICADO}

La cardiopatía isquémica ocasiona el mayor número de muertes cardiovasculares y, dentro de ella, el infarto agudo de miocardio (IAM) es la más frecuente.

Aunque la mayoría de los pacientes responden al tratamiento médico, otros llegan a necesitar intubación y ventilación mecánica. Los factores de riesgo asociados a la necesidad de ventilación mecánica según el estudio ARIAM se encuentran detallados en la Tabla $\mathbf{2}^{12,13}$. Otras causas de intubación fueron shock cardiogénico, edema agudo pulmonar y arritmias de difícil control.

Aquellos pacientes con IAM complicado que requieren ventilación mecánica pueden sufrir daño pulmonar e inducir o perpetuar la lesión pulmonar aguda (LPA) si no son manejados adecuadamente. Este fenómeno (lesión pulmonar asociada o inducida por el respirador o VILI) se produce tanto en pulmones sanos, como en aquellos previamente dañados. Ello quedó demostrado en los estudios publicados por el Acute Respiratory Distress Syndrome Network (ARDSnet), que evidenciaron una reducción relativa del riesgo de muerte del $22 \%$ en aquellos pacientes ventilados con una estrategia respiratoria protectora del pulmón.

Los mecanismos de producción de lesión alveolar en pacientes isquémicos bajo ventilación mecánica son ${ }^{14,15}$ :

Daño pulmonar inducido por fracciones inspiradas de oxígeno elevadas: Clásicamente se describe un efecto tóxico sobre el pulmón por el empleo de unaFiO2 elevada durante periodos prolongados de tiempo, aunque las únicas lesiones que se han podido demostrar son los fenómenos de atelectasia, con el consiguiente efecto shunt y una bronquitis hiperóxica.

Daño pulmonar inducido por presión excesiva en la vía aérea (barotrauma) y sobredistensión pulmonar (volutrauma): Ios pacientes que son ventilados con elevadas presiones alveolares (presión plateau o meseta mayor de $30 \mathrm{cmH}_{2} \mathrm{O}$ ) presentaron lesiones consistentes en un importante edema tanto perivascular como alveolar, incrementando el deterioro hemodinámico secundario al edema agudo no cardiogénico ${ }^{16}$. Por otro lado, los volúmenes pulmonares elevados incrementan la permeabilidad alveolar, por lo que se recomienda programar 8 $\mathrm{ml} / \mathrm{kg} /$ peso ideal en pacientes sin daño pulmonar y $5-7 \mathrm{ml} / \mathrm{kg} /$ peso ideal en pacientes con daño pulmonar.

Daño pulmonar inducido por bajo volumen total (atelectrauma): el repetido colapso y apertura cíclica del alvéolo genera daño pulmonar por lo que el empleo de la PEEP por encima del punto de colapso alveolar mejora la distensibilidad y evita el cizallamiento alveolar. Se recomienda utilizar niveles adecuados de PEEP para no modificar el estado hemodinámico del paciente y siempre en lo posible optimizar la precarga. Daño pulmonar inducido por factores inflamatorios (biotrauma): existen mecanismos celulares y humorales que ocasionan reacciones bioquímicas e inflamatorias que finalizan en lesión pulmonar. Esta elevada producción local de citocinas favorece una respuesta inflamatoria local y sistémica, translocación bacteriana, edema pulmonar y falla orgánica múltiple en estadios avanzados. Existe evidencia de que la ventilación protectora (bajos volúmenes y PEEP elevado) se asocia a una importante reducción en la mortalidad.

Existen otros parámetros ventilatorios que también juegan un papel importante en el desarrollo de daño pulmonar, como el flujo constante, variable que se relaciona con el incremento de sobredistensión pulmonar en las zonas no dependientes, razón que justifica el uso del flujo desacelerado. También el ciclado frecuente (>25 por min) se relaciona con mayor daño pulmonar.

La tasa de mortalidad en pacientes con IAM complicado y AVM sigue siendo alta y varía de 13,5\% al 28\% para pacientes hospitalizados en la unidad de cuidados intensivos, siendo la fracción de eyección ventricular izquierda $<40 \%$ y el shock cardiogénico predictores independientes de mortalidad después de un infarto ${ }^{17}$

\section{CONCLUSIÓN}

La falla cardíaca aguda cursa con síntomas que ponen en riesgo de vida a los pacientes, con trastornos graves en la oxigenación, que requieren un soporte vital avanzado para lograr la compensación cardiorespiratoria. La intubación orotraqueal en general es un procedimiento de urgencia en estas situaciones, sin embargo deben tomarse 
los recaudos necesarios, con una preparación adecuada del paciente y además contar con un plan alternativo ante el fallo en la intubación. La elección de los fármacos (relajantes musculares y sedoanalgesia) en dicho procedimiento y durante la ventilación mecánica requiere del conocimiento de sus efectos hemodinámicos a fin de evi- tar mayor compromiso cardiovascular del paciente. Asimismo, es de vital importancia evaluar las respuestas fisiopatológicas que ejerce la presión positiva en el paciente cardiópata, por lo que la programación del ventilador debe ajustarse acorde al monitoreo de la mecánica respiratoria, los gases arteriales y el estado hemodinámico.

\section{BIBLIOGRAFÍA}

1. Adams KF, Fonarow GC, Emerman CL, Lelemtel TH, Costanzo MR Abraham WT et al. Characteristics and outcome of patients hospitalized for heart failure in the United States: Rationale, design, and preliminary observations from the first 100 000 cases in the Acute Decompensated Heart Failure National Registry (ADHERE). Am Heart J 2005;149(2):209-16

2. Nieminen MS, Brutsaert D, Dickstein K, Drexler H, Follath F, Harjola VP, et Al. Euro HeartFailureSurvey II (EHFS II): A survey on hospitalized acute heart failure patients: Description of population. Eur Heart J 2006;27(22):2725-36.

3. Castillo Costa Y, Mauro V. Shock cardiogénico. En Sociedad Argentina de Terapia Intensiva, Terapia Intensiva (2008, cuarta edición, 574-583). Argentina: Editorial Panamericana.

4. Jensen AG, Callensen T, Hagemo JS, Hreinsson K, Lund V, Nordmark J, et al. Scandinavian clinical practice guidelines on general anaesthesia for emergency situations. Acta Anaesthesiol Scand 2010;54(8):922-50.

5. Altermatt FR, Munoz HR, Delfino AE, Cortinez LI. Preoxygenation in the obese patient: effects of position on tolerance to apnoea. Br J Anaesth 2005:95(5):706-9.

6. Stoelting RK, Hillier S. Pharmacology and Physiology in Anesthetic Practice (2005, Cuarta edición, 144). Estados Unidos: Editorial Lippincott Williams and Wilkins.

7. Cork RC, Weiss JL, Hameroff SR, Bentley J. Fentanyl preloading for rapid-sequence induction of anesthesia. Anesth Analg 1984;63(1):60-4

8. Boralessa H, Senior DF, Whitwam JG. Cardiovascular response to intubation. A comparative study of thiopentone and midazolam. Anaesthesia 1983;38(7):623-7. Levitt MA. A prospective, randomized trial of BiPAP in severe acute congestive heart failure. J Emerg Med 2001;21(4):363-9.
9. Mehta S, Jay GD, Woolard RH, Hipona RA, Connolly EM, Drinkwine JH, et al. Randomized, prospective trial of bilevel versus continuous positive airway pressure in acute pulmonary edema. Crit Care Med 1997;25(4):620-8.

10. Masip J, Betbesé AJ, Páez J, Vecilla F, Cañizares R, Padró J, et Al. Non invasive pressure support ventilation versus conventional oxygentherapy in acute cardiogenic pulmonary oedema: a randomized trial. Lancet 2000;356(9248): 2126-32.

11. Pinsky MR. The effects of mechanical ventilation on the cardiovascular system Crit Care Clin 1990;6(3):663-78

12. Marini JJ, Gattinoni L. Ventilatory management of acute respiratory distress syndrome: a consensus of two. Crit Care Med 2004;32(1):250-5.

13. Esteban A, Anzueto A, Frutos F, Alia I, Brochard L, Stewart T et al. Characteristics and outcomes in adult patients receiving mechanical ventilation. A 28-day international study. JAMA 2002:287(3):345-55.

14. Brower RG, Matthay MA, Morris A, Schoenfeld D, Thompson BT, Wheeler A, et al. The Acute Respiratory Distress Syndrome Network. Ventilation with lower tidal volumes as compared with traditional tidal volumes for acute lung injury and the acute respiratory distress syndrome. N Engl J Med 2000;342(18):1301-8.

15. Dreyfuss D, Soler P, Basset G, Saumom G. High inflation pressure pulmonary edema. Respective effects of high airway pressure, high tidal volume, and positive end-expiratory pressure. Am J Respir Crit Care Med 1988:137(5):1159-64.

16. Villalobos SJA, Montes OMA, Camacho MLA. Ventilación mecánica en pacientes con cardiopatía aguda. Rev Mex Cardiol 2011;22(2):96-107. 